\title{
Semimodularity and the Logic of Quantum Mechanics*
}

\author{
James C. T. Pool \\ Institut de Physique Théorique, Université de Genève \\ Applied Mathematics Division, Argonne National Laboratory
}

Received March 25, 1968

\begin{abstract}
If $(\mathscr{E}, \mathscr{S}, P, \Omega)$ is an event-state-operation structure, then the events form an orthomodular ortholattice $\left(\mathscr{E}, \leqq,^{\prime}\right)$ and the operations, mappings from the set of states $\mathscr{S}$ into $\mathscr{S}$, form a Baer ${ }^{*}$-semigroup $\left(S_{\Omega}, \circ,{ }^{*},{ }^{\prime}\right)$. Additional axioms are adopted which yield the existence of a homomorphism $\theta$ from $\left(S_{\Omega}, \circ, *,{ }^{\prime}\right)$ into the Baer *-semigroup $\left(S(\mathscr{E}), \circ,{ }^{*},{ }^{\prime}\right)$ of residuated mappings of $\left(\mathscr{E}, \leqq,^{\prime}\right)$ such that $x \in S_{\Omega}$ maps states while $\theta_{x} \in S(\mathscr{E})$ maps supports of states. If $\left(\mathscr{E}, \leqq,^{\prime}\right)$ is atomic and there exists a correspondence between atoms and pure states, then the existence of $\theta$ provides the result: $\left(\mathscr{E}, \leqq,^{\prime}\right)$ is semimodular if and only if every operation $x \in S_{\Omega \Omega}$ is a pure operation (maps pure states into pure states).
\end{abstract}

\section{Introduction}

The theory of orthomodular ortholattices provides the mathematical constructs for the quantum logic approach to the foundations of quantum physics. A role for the theory of Baer *-semigroup, a mathematical theory closely related to the theory of orthomodular ortholattices, was exhibited in [15]. The definitions and terminology introduced in [15] will be utilized in this paper without further explanation. If $(\mathscr{E}, \mathscr{S}, P, \Omega)$ is an event-state-operation structure, then $\left(\mathscr{E}, \leqq,^{\prime}\right)$ is an orthomodular ortholattice and $\left(S_{\Omega}, \circ,{ }^{*},{ }^{\prime}\right)$ is a Baer *-semigroup such that $p \in \mathscr{E}$ $\rightarrow \Omega_{p} \in P^{\prime}\left(S_{\Omega}\right)$ is an isomorphism of $\left(\mathscr{E}, \leqq,^{\prime}\right)$ onto the orthomodular ortholattice $\left(P^{\prime}\left(S_{\Omega}\right),,^{\prime}\right)$ of closed projections in $S_{\Omega}$. Each $x \in S_{\Omega}$ is a mapping, $x: \mathscr{D}_{x} \rightarrow \mathscr{R}_{x}$, with domain $\mathscr{D}_{x}$ and range $\mathscr{R}_{x}$ contained in $\mathscr{S}$.

The connection between the theories of orthomodular ortholattices and Baer *-semigroups includes the following: if $\left(L, \leqq,^{\prime}\right)$ is any orthomodular ortholattice, then there exists a Baer *-semigroup $\left(S(L), \circ, *,{ }^{\prime}\right)$ where $S(L)$ is a set of mappings of $L$ into $L$ and there exists an injective mapping $j: L \rightarrow S(L)$. Section I is devoted to a discussion of $S(\mathscr{E})$ for the orthomodular ortholattice $\left(\mathscr{E}, \leqq,^{\prime}\right)$. In particular, the relation of $\left(S(\mathscr{E}), \circ, *,{ }^{\prime}\right)$ to the Baer ${ }^{*}$-semigroup $\left(S_{\Omega}, \circ,{ }^{*},{ }^{\prime}\right)$ of operations will be exhibited.

* Supported in part by the United States Atomic Energy Commission and in part by the Fonds National Suisse. 
One goal of the quantum logic approach to the foundations of quantum physics is to augment the axioms of an event-state structure to obtain an axiomatic characterization of von Neumann's Hilbert space model for quantum mechanics : an axiomatic characterization where each axiom has a plausible physical interpretation. The currently available "concrete representation theorems" (the identification of $(\mathscr{E}, \leqq$, ') with a lattice of subspaces of a vector space) require the following hypothesis: $\left(\mathscr{E}, \leqq,^{\prime}\right)$ is semimodular. The correspondence between $\left(S_{\Omega}, \circ,{ }^{*},{ }^{\prime}\right)$ and $(S(\mathscr{E}), \circ, *, ')$ will be utilized to obtain a direct phenomenological interpretation of the semimodularity of $\left(\mathscr{E}, \leqq,^{\prime}\right)$ in Section III. The setting for the investigation of semimodularity will be developed in Section II.

Definitions and theorems relating to orthomodular ortholattices and Baer *-semigroups were presented in the Appendix of [15]. Additional definitions and theorems concerning residuated mappings, atomicity, and semimodularity are included in an Appendix to this paper.

\section{A Role for Residuated Mappings of $\left(\mathscr{E}, \leqq,^{\prime}\right)$}

The set $S(L)$ of residuated mappings of any orthomodular ortholattice $\left(L, \leqq,^{\prime}\right)$ admits the structure of a Baer *-semigroup $\left(S(L), \circ,{ }^{*},{ }^{\prime}\right)$; moreover, the orthomodular ortholattice $\left(P^{\prime}(S(L)), \leqq,^{\prime}\right)$ of closed projections in $S(L)$ is isomorphic to $\left(L, \leqq,^{\prime}\right)$. Therefore, if $(\mathscr{E}, \mathscr{S}, P, \Omega)$ is an event-state-operation structure, then the residuated mappings of the orthomodular ortholattice $\left(\mathscr{E}, \leqq,^{\prime}\right)$ form a Baer *-semigroup $\left(S(\mathscr{E}), \circ,{ }^{*},,^{\prime}\right)$. The question arises whether this Baer*-semigroup is related to the Baer*-semigroup $\left(S_{\Omega}, \circ, *,,^{\prime}\right)$ of operations in a phenomenologically interpretable way. The answer to this question requires a review of the concept of support of a state.

Definition I.1. Let $(\mathscr{E}, \mathscr{S}, P)$ be an event-state structure.

(a) If $\alpha \in \mathscr{S}$, then $\mathscr{E}_{0}(\alpha)$ and $\mathscr{E}_{1}(\alpha)$ are the subsets of $\mathscr{E}$ defined by

$$
\begin{aligned}
& \mathscr{E}_{0}(\alpha)=\{p \in \mathscr{E}: P(p, \alpha)=0\} \\
& \mathscr{E}_{1}(\alpha)=\{p \in \mathscr{E}: P(p, \alpha)=1\} .
\end{aligned}
$$

(b) If $\alpha \in \mathscr{S}$, then $p \in \mathscr{E}$ is a support of $\alpha$ provided:

for $q \in \mathscr{E}, P(q, \alpha)=0$ if and only if $q \perp p$.

The validity of the following assertions is evident.

Theorem 1.1. Let $(\mathscr{E}, \mathscr{S}, P)$ be an event-state structure.

(a) If $\alpha \in \mathscr{S}$ and $p \in \mathscr{E}$, then the following are equivalent:

i) $p$ is a support of $\alpha$,

ii) $\mathscr{E}_{0}(\alpha)=\{q \in \mathscr{E}: q \perp p\}$,

iii) $\mathscr{E}_{1}(\alpha)=\{q \in \mathscr{E}: p \leqq q\}$,

iv) $p$ is the least element of the subset $\mathscr{E}_{1}(\alpha)$ of $\mathscr{E}$.

(b) If $\alpha \in \mathscr{S}$, then there exists at most one $p \in \mathscr{E}$ such that $p$ is a support of $\alpha$. 
Definition I.2. If $(\mathscr{E}, \mathscr{S}, P)$ is an event-state structure and $\alpha \in \mathscr{S}$, then the support of $\alpha$, provided it exists, is denoted by $p_{\alpha}$.

The investigation of the role of $S(\mathscr{E})$ will involve the adoption of another axiom to supplement the seven axioms for event-state structures presented in [15].

Axiom I.8. (a) If $\alpha \in \mathscr{S}$, then the support $p_{\alpha}$ of $\alpha$ exists.

(b) If $p \in \mathscr{E}$ and $p \neq 0$, then there exists an $\alpha \in \mathscr{S}$ such that $p$ is the support of $\alpha$.

Axiom I.8 is valid for von Neumann's Hilbert space model of quantum mechanics; indeed, this axiom is valid for a wide class of event-state structures [21].

Example I.1. (See Example I.1 of [15]). Let $(\mathscr{P}(H), \mathscr{S}, P)$ be the event-state structure for von Neumann's Hilbert space model of quantum mechanics. If $\alpha \in \mathscr{S}$, then the support of $\alpha$ is the operator-theoretic support projection of the density operator $D_{\alpha}$ corresponding to $\alpha$, since $\operatorname{Tr}$ is a faithful normal trace on the von Neumann algebra $\mathscr{L}_{c}(H)$ of all continuous linear operators on $H$ (see, for example, [4], [5] and [16]). The operator-theoretic support projection of $D_{\alpha}$ is the projection on the orthogonal complement of the null space of $D_{\alpha}$; hence, in terms of the Baer *-semigroup $\left(\mathscr{L}_{c}(H), \circ,{ }^{*},,^{\prime}\right)$ (see the Appendix of [15]), the support of $\alpha$ is $\left(D_{\alpha}\right)^{\prime \prime}$.

Theorem 1.2. Let $(\mathscr{E}, \mathscr{S}, P, \Omega)$ be an event-state-operation structure satisfying Axiom I.8. If $\mathrm{p} \in \mathscr{E}, \alpha \in \mathscr{S}, P(p, \alpha) \neq 0$ and $\beta=\Omega_{p} \alpha$, then the support $p_{\beta}$ of $\beta$ and the support $p_{\alpha}$ of $\alpha$ satisfy

$$
p_{\beta} \leqq\left(p_{\alpha} \vee p^{\prime}\right) \wedge p
$$

Proof. $p_{\alpha}$ is the support of $\alpha$ and $p_{\alpha}^{\prime} \perp p_{\alpha}$; hence, $P\left(p_{\alpha}^{\prime}, \alpha\right)=0$ by the defining property of support. Since $p_{\alpha}^{\prime} \wedge p \leqq p_{\alpha}$, it follows that

$$
0 \leqq P\left(p_{\alpha}^{\prime} \wedge p, \alpha\right) \leqq P\left(p_{\alpha}^{\prime}, \alpha\right)=0
$$

and, since $p_{\alpha}^{\prime} \wedge p \leqq p$, Axiom II.6 of [15] implies

$$
P\left(p_{\alpha}^{\prime} \wedge p, \Omega_{p} \alpha\right)=\frac{P\left(p_{\alpha}^{\prime} \wedge p, \alpha\right)}{P(p, \alpha)}
$$

hence, $P\left(p_{\alpha}^{\prime} \wedge p, \Omega_{p} \alpha\right)=0$. Since $\beta=\Omega_{p} \alpha$, one has

$$
P\left(p_{\alpha} \vee p^{\prime}, \beta\right)=1-P\left(p_{\alpha}^{\prime} \wedge p, \beta\right)=1 .
$$

By assertion a) of Theorem I.1, $P\left(p_{\alpha} \vee p^{\prime}, \beta\right)=1$ implies $p_{\beta} \leqq p_{\alpha} \vee p^{\prime}$. Axiom II.2 of [15] asserts $P\left(p, \Omega_{p} \alpha\right)=1$; hence, $P(p, \beta)=1$, since $\beta=\Omega_{p} \alpha$, and $p_{\beta} \leqq p$ again by Theorem I.1. Therefore, $p_{\beta} \leqq p_{\alpha} \vee p^{\prime}$ and $p_{\beta} \leqq p$; hence, $p_{\beta} \leqq\left(p_{\alpha} \vee p^{\prime}\right) \wedge p$. Q.E.D.

The assertion of Theorem I.2 may be expressed in terms of residuated mappings as follows: if $p \in \mathscr{E}, \alpha \in \mathscr{D}_{p}$ and $\beta=\Omega_{p} \alpha$, then $p_{\beta} \leqq \phi_{p}\left(p_{\alpha}\right)$, where $\phi_{p}$ is the following residuated mapping of $\mathscr{E}$ into $\mathscr{E}$ :

$$
\phi_{p}(q)=\left(q \vee p^{\prime}\right) \wedge p, \quad q \in \mathscr{E} .
$$


The example of von Neumann's Hilbert space model of quantum mechanics and the case of a compatible logic provide stronger results than Theorem I.2.

Example I.2. For the event-state-operation structure $(\mathscr{P}(H), \mathscr{S}, P, \Omega)$ (see Example II.1 of [15]), $\Omega$ is defined as follows: if $P \in \mathscr{P}(H), \alpha \in \mathscr{S}$, $P(P, \alpha) \neq 0$, and $D_{\alpha}$ is the density operator for $\alpha$, then $\beta=\Omega_{p} \alpha$ is the state with density operator $D_{\beta}$,

$$
D_{\beta}=\frac{P D_{\alpha} P}{\operatorname{Tr}\left(D_{\alpha} P\right)} .
$$

The supports of $\alpha$ and $\beta$ are $P_{\alpha}=\left(D_{\alpha}\right)^{\prime \prime}$ and $P_{\beta}=\left(D_{\beta}\right)^{\prime \prime}$, respectively, in terms of the Baer $*_{\text {-semigroup }}\left(\mathscr{L}_{c}(H), \circ,{ }^{*},{ }^{\prime}\right)$. The support $\left(D_{\beta}\right)^{\prime \prime}$ coincides with $\left(P D_{\alpha} P\right)^{\prime \prime}$, since the positive number $\left(T_{r}\left(D_{\alpha} P\right)\right)^{-1}$ is immaterial for supports. $D_{\alpha}$ is a positive operator on $H$; hence, there exists a $T_{\alpha} \in \mathscr{L}_{c}(H)$ such that $D_{\alpha}=T_{\alpha}^{*} T_{\alpha}$. Consequently, Theorem A.5 of the Appendix asserts

$$
\left(D_{\beta}\right)^{\prime \prime}=\left(P D_{\alpha} P\right)^{\prime \prime}=\left(\left(D_{\alpha}\right)^{\prime \prime} \vee P^{\prime}\right) \wedge P .
$$

Therefore, the inequality of the conclusion of Theorem I.2 is replaced by an equality

$$
P_{\beta}=\left(P_{\alpha} \vee P^{\prime}\right) \wedge P
$$

for the special case of von Neumann's Hilbert space model for quantum physics.

Theorem I.3. Let $(\mathscr{E}, \mathscr{S}, P, \Omega)$ be an event-state-operation structure such that

i) Axiom $I .8$ is satisfied, and

ii) if $p, q \in \mathscr{E}$, then $p \mathrm{C} q$.

If $p \in \mathscr{E}, \alpha \in \mathscr{S}, P(p, \alpha) \neq 0$ and $\beta=\Omega_{p} \alpha$, then

$$
p_{\beta}=p_{\alpha} \wedge p=\left(p_{\alpha} \vee p^{\prime}\right) \wedge p .
$$

Proof. Because of hypothesis ii), the relation of orthogonality may be characterized as follows (see, for example, [14]): for $p, q \in \mathscr{E}, p \perp q$ if and only if $p \wedge q=0$. If $q \in \mathscr{E}$, then $p \subset q$ and

$$
P(q, \beta)=P\left(q, \Omega_{p} \alpha\right)=\frac{P(q \wedge p, \alpha)}{P(p, \alpha)}
$$

by Theorem IV.1 of [15]; hence, for $q \in \mathscr{E}, P(q, \beta)=0$ if and only if $P(q \wedge p, \alpha)=0 . P(q \wedge p, \alpha)=0$ if and only if $q \wedge p \perp p_{\alpha}$ by the definition of the support of $\alpha . q \wedge p \perp p_{\alpha}$ if and only if $q \perp p \wedge p_{\alpha}$, since $(q \wedge p) \wedge p_{\alpha}$ $=q \wedge\left(p \wedge p_{\alpha}\right)$ and $\perp$ has the above characterization. Consequently, for $q \in \mathscr{E}, P(q, \beta)=0$ if and only if $q \perp p \wedge p_{\alpha}$. This, however, is the defining property for the support $p_{\beta}$ of $\beta$; hence, $p_{\beta}=p_{\alpha} \wedge p$. Since $p \subset p_{\alpha}$, the characteristic properties of the relation $C$ (see Section III of [15]) imply $\left(p_{\alpha} \vee p^{\prime}\right) \wedge p=p_{\alpha} \wedge p$. Q.E.D. 
The following axiom for event-state-operation structures is, therefore, motivated by the general result of Theorem I.2 and the results of the special cases of Example I.2 and Theorem I.3.

Axiom II.8. If $p \in \mathscr{E}, \alpha \in \mathscr{S}, P(p, \alpha) \neq 0, \beta=\Omega_{p} \alpha$, and the supports $p_{\alpha}$ and $p_{\beta}$ of $\alpha$ and $\beta$ exist, then

$$
p_{\beta}=\phi_{p}\left(p_{\alpha}\right)=\left(p_{\alpha} \vee p^{\prime}\right) \wedge p .
$$

Theorem 1.4. Let $(\mathscr{E}, \mathscr{S}, P, \Omega)$ be an event-state-operation structure satisfying Axioms $I .8$ and $I I .8$ with $x \in S_{\Omega}, p_{1}, p_{2}, \ldots, p_{n} \in \mathscr{E}$, and $x=\Omega_{p_{1}} \circ \Omega_{p_{2}} \circ \cdots \circ \Omega_{p_{n}}$.

a) If $\alpha \in \mathscr{D}_{x}$, then

$$
p_{x \alpha}=\phi_{p_{1}} \circ \phi_{p_{2}} \circ \cdots \circ \phi_{p_{n}}\left(p_{\alpha}\right)
$$

b) $\alpha \in C \mathscr{D}_{x}$ if and only if

$$
\phi_{p_{1}} \circ \phi_{p_{2}} \circ \cdots \circ \phi_{p_{n}}\left(p_{\alpha}\right)=0 \text {. }
$$

c) $\alpha \in C \mathscr{D}_{x}$ if and only if

$$
p_{\alpha} \leqq\left(\phi_{p_{1}} \circ \phi_{p_{2}} \circ \cdots \circ \phi_{p_{n}}\right)^{*}(1)^{\prime} .
$$

d) $q_{x}=\left(\phi_{p_{1}} \circ \phi_{p_{2}} \circ \cdots \circ \phi_{p_{n}}\right)^{*}(1)^{\prime}$.

Proof. Assertion a) follows from Axiom II. 8 by induction on $n$. Let $p_{n+1}=1$. The following characterization of $C \mathscr{D}_{a}$ was obtained in Section II of [15]: $\alpha \in C \mathscr{D}_{x}$ if and only if there exists an $i, 1 \leqq i \leqq n$, such that

$$
P\left(p_{j}, \Omega_{p_{j+1}} \circ \cdots \circ \Omega_{p_{n}} \circ \Omega_{p_{n+1}} \alpha\right) \neq 0
$$

for $i \leqq j \leqq n$ and

$$
P\left(p_{i}, \Omega_{p_{i+1}} \circ \cdots \circ \Omega_{p_{n}} \circ \Omega_{p_{n+1}} \alpha\right)=0 .
$$

Since the supports of the states involved in these two expressions may be determined by utilizing a), this characterization of $C \mathscr{D}_{x}$ yields the following: $\alpha \in C \mathscr{D}_{x}$ if and only of there exists an $i, 1 \leqq i \leqq n$, such that

for $i<j \leqq n$ and

$$
\phi_{p_{j+1}} \circ \cdots \circ \phi_{p_{n}} \circ \phi_{p_{n+1}}\left(p_{\alpha}\right) \perp p_{j}
$$

$$
\phi_{p_{i+1}} \circ \cdots \circ \phi_{p_{n}} \circ \phi_{p_{n+1}}\left(p_{\alpha}\right) \not p_{i} .
$$

For any $p, q \in \mathscr{E}, \phi_{p}(q)=0$ if and only if $p \perp q$. Hence, the characterization of $C \mathscr{D}_{x}$ may be expressed as follows: $\alpha \in C \mathscr{D}_{x}$ if and only if $\phi_{p_{1}} \circ \phi_{p_{2}} \circ \cdots \circ \phi_{p_{n}}\left(p_{\alpha}\right)=0$. Thus, assertion b) is established. For any $q \in \mathscr{E}$ and $\phi \in S(\mathscr{E}), \phi(q)=0$ if and only if $q \leqq \phi^{*}(1)^{\prime}$; hence, assertion c) follows immediately from b). Because of the properties of $p_{\alpha}$, c) asserts

$$
C \mathscr{D}_{x}=\left\{\alpha \in \mathscr{S}: P\left(\left(\phi_{p_{1}} \circ \phi_{p_{2}} \circ \cdots \circ \phi_{p_{n}}\right)^{*}(1)^{\prime}, \alpha\right)=1\right\} \text {. }
$$

Since $q_{x}$ is the unique element of $\mathscr{E}$ such that

$$
C \mathscr{D}_{x}=\mathscr{S}_{1}\left(q_{x}\right)=\left\{\alpha \in \mathscr{S}: P\left(q_{x}, \alpha\right)=1\right\}
$$

(see Definition II.3 of [15]), assertion d) is valid. Q.E.D. 
Theorem I.5. Let $(\mathscr{E}, \mathscr{S}, P, \Omega)$ be an event-state-operation structure satisfying Axioms $I .8$ and $I I .8$.

a) The mapping $\theta: S_{\Omega} \rightarrow S(\mathscr{E})\left(x \in S_{\Omega} \rightarrow \theta_{x} \in S(\mathscr{E})\right)$ defined as follows is well-defined: if $x \in S_{\Omega}$, then select $p_{1}, p_{2}, \ldots, p_{n} \in \mathscr{E}$ such that $x=\Omega_{p_{1}} \circ$ $\circ \Omega_{p_{2}} \circ \cdots \circ \Omega_{p_{n}}$ and define $\theta_{x}$ by

$$
\theta_{x}=\phi_{p_{1}} \circ \phi_{p_{2}} \circ \cdots \circ \phi_{p_{n}} \text {. }
$$

b) $\theta$ is a homomorphism of the Baer *-semigroup $\left(S_{\Omega}, \circ, *,{ }^{*}\right)$ of operations into the Baer *-semigroup $\left(S(\mathscr{E}), \circ, *,{ }^{\prime}\right)$ of residuated mappings of $\left(\mathscr{E}, \leqq,^{\prime}\right)$.

c) If $x \in S_{\Omega}$ and $\alpha \in \mathscr{D}_{x}$, then

$$
p_{x \alpha}=\theta_{x}\left(p_{\alpha}\right)
$$

d) If $x \in S_{\Omega}$ and $\alpha \in \mathscr{S}$, then the following are equivalent:

i) $\alpha \notin \mathscr{D}_{x}$,

ii) $\theta_{x}\left(p_{\alpha}\right)=0$,

iii) $p_{\alpha} \leqq \theta_{x}^{*}(\mathbf{1})^{\prime}$.

Proof. $\theta: S_{\Omega} \rightarrow S(\mathscr{E})$ is well-defined provided: if $x \in S_{\Omega}, p_{1}, p_{2}, \ldots, p_{n}$, $q_{1}, q_{2}, \ldots, q_{m} \in \mathscr{E}$, and

then

$$
x=\Omega_{p_{1}} \circ \Omega_{p_{2}} \circ \cdots \circ \Omega_{p_{n}}=\Omega_{q_{1}} \circ \Omega_{q_{2}} \circ \cdots \circ \Omega_{q_{m}},
$$

$$
\phi_{p_{1}} \circ \phi_{p_{2}} \circ \cdots \circ \phi_{p_{n}}=\phi_{q_{1}} \circ \phi_{q_{2}} \circ \cdots \circ \phi_{q_{m}}
$$

that is, if $p \in \mathscr{E}$, then

$$
\phi_{p_{1}} \circ \phi_{p_{2}} \circ \cdots \phi_{p_{n}}(p)=\phi_{q_{1}} \circ \phi_{q_{2}} \circ \cdots \phi_{q_{m}}(p) \text {. }
$$

Both sides of (I) are equal to 0 , if $p=0$. If $p \in \mathscr{E}$ and $p \neq 0$, then there exists an $\alpha \in \mathscr{S}$ such that $p$ is the support $p_{\alpha}$ of $\alpha, p=p_{\alpha}$, by Axiom I.8. If $\alpha \in \mathscr{D}_{x}$, then both sides of (I) are equal to the support of $x \alpha$ by assertion a) of Theorem I.4. If $\alpha \notin \mathscr{D}_{x}$, then both sides of (I) are equal to 0 by assertion b) of Theorem I.4. Consequently, $\theta$ is well-defined.

$\theta$ obviously preserves $\circ$ and $*$,

$$
\theta_{x \circ y}=\theta_{x} \circ \theta_{y}, \quad \theta_{x^{*}}=\left(\theta_{x}\right)^{*}
$$

for $x, y \in S_{\Omega}$. If $x \in S_{\Omega}, p_{1}, p_{2}, \ldots, p_{n} \in \mathscr{E}$ and $x=\Omega_{p_{1}} \circ \Omega_{p_{2}} \circ \cdots \circ \Omega_{p_{n}}$, then $x^{\prime}=\Omega_{q_{x}}$ (see Definition II.3 of [15]). By the definition of 'for $\left(S(\mathscr{E}), \circ, *,{ }^{\prime}\right),\left(\theta_{x}\right)^{\prime}=\phi_{\alpha}$ where $q \in \mathscr{E}$ is given by

$$
q=\theta_{x}^{*}(1)^{\prime}=\left(\phi_{p_{1}} \circ \phi_{p_{2}} \circ \cdots \circ \phi_{p_{n}}\right)^{*}(1)^{\prime} \text {. }
$$

d) of Theorem I.4 asserts that $q=q_{x}$; hence, $\left(\theta_{x}\right)^{\prime}=\phi_{q_{x}}$. Since $x^{\prime}=\Omega_{q_{x}}$, $\left(\theta_{x}\right)^{\prime}=\theta_{x^{\prime}}$ and $\theta$ preserves '.

Assertion c) is an immediate consequence of assertion a) of Theorem I.4 while assertion d) is an immediate consequence of b) and c) of Theorem I.4. Q.E.D.

15 Commun. math. Phys., Vol. 9 
Consequently, if $(\mathscr{E}, \mathscr{S}, P, \Omega)$ is an event-state-operation structure satisfying Axioms I.8 and II.8, then, for each element $x$ of the Baer *-semigroup $\left(S_{\Omega}, \circ,{ }^{*},{ }^{\prime}\right)$ of operations, there is an element $\theta_{x}$ of the Baer $*_{\text {-semigroup }}\left(S(\mathscr{E}), \circ,{ }^{*},{ }^{\prime}\right)$ of residuated mappings of $\left(\mathscr{E}, \leqq,^{\prime}\right)$. $x$ maps states while $\theta_{x}$ maps supports of states; specifically, if $\alpha \in \mathscr{D}_{x}$ then the support $p_{\alpha}$ of the state $\alpha$ is mapped into the support $p_{x \alpha}$ of the state $x \propto$ by $\theta_{x}$,

$$
p_{x \alpha}=\theta_{x}\left(p_{\alpha}\right) .
$$

\section{Atoms and Pure States}

The purpose of this section is to augment the axioms of an eventstate structure to provide a setting for investigating the role of semimodularity.

Definition II.1. Let $(\mathscr{E}, \mathscr{S}, P)$ be an event-state structure.

a) If $\alpha_{1}, \alpha_{2}, \ldots, \in \mathscr{S}, t_{1}, t_{2}, \ldots, \in[0,1]$, and $\sum_{i} t_{i}=1$, then the unique $\alpha \in \mathscr{S}$ such that

$$
P(p, \alpha)=\sum_{i} t_{i} P\left(p, \alpha_{i}\right),
$$

for all $p \in \mathscr{E}$ (see Axioms I.6 and I.7 of [15]) is denoted by

$$
\alpha=\sum_{i} t_{i} \alpha_{i}
$$

and called the mixture of $\alpha_{1}, \alpha_{2}, \ldots$ with respective weights $t_{1}, t_{2}, \ldots$

b) A state $\alpha \in \mathscr{S}$ is pure provided: if $t \in(0,1), \alpha_{1}, \alpha_{2} \in \mathscr{S}$, and

$$
\alpha=t \alpha_{1}+(1-t) \alpha_{2},
$$

then $\alpha_{1}=\alpha_{2}$; otherwise, $\alpha$ is mixed. The set of all pure states is denoted by $\tilde{S .}$

c) The set of all atoms in $\mathscr{E}$ is denoted by $\widetilde{\mathscr{E}}$.

For a general event-state structure, $\mathscr{E}$ is not atomic; indeed there may be no atoms in $\mathscr{E}$ (for the definitions of atom and atomic, see the Appendix). Furthermore, there may exist no pure states in $\mathscr{S}$. An additonal axiom is necessary [12].

Axiom 1.9. a) If $p \in \mathscr{E}$ and $p \neq 0$, then there exists a pure state $\alpha \in \mathscr{S}$ such that $P(p, \alpha)=1$.

b) $\alpha \in \mathscr{S}$ is a pure state if and only of there exists a $p \in \mathscr{E}$ such that, for $\beta \in \mathscr{S}, P(p, \beta)=1$ is equivalent to $\beta=\alpha$.

If $p \in \mathscr{E}$ and $p \neq 0$, then there exists an $\alpha \in \mathscr{S}$ such that $P(p, \alpha)=1$ by assertion f) of Theorem I.1 of [15]. Part a) of Axiom I.9 asserts that this state may be selected to be a pure state. Part b) of Axiom I.9 asserts that a state is pure if and only if it may be prepared (see Theorem III.1) and identified by observing a single event. 
Theorem II.1. If $(\mathscr{E}, \mathscr{S}, P)$ is an event-state structure satisfying Axiom I.8, then the following are equivalent statements:

a) $(\mathscr{E}, \mathscr{S}, P)$ satisfies Axiom $I .9$,

b) $(\mathscr{E}, \mathscr{S}, P)$ satisfies the following:

i) if $p \in \mathscr{E}$ and $p \neq 0$, then there exists a pure state $\alpha \in \mathscr{S}$ such that $P(p, \alpha)=1$, and

ii) $\alpha \in \mathscr{S}$ is a pure state if and only if $p_{\alpha}$ is an atom and $\alpha$ is the unique state in $\mathscr{S}$ with support equal to $p_{\alpha}$; and

c) $(\mathscr{E}, \mathscr{S}, P)$ satisfies the following:

i) $\left(\mathscr{E}, \leqq,^{\prime}\right)$ is atomic and

ii) there exists a mapping $p \rightarrow \alpha_{p}$ of the set of atoms, $\tilde{\mathscr{E}}$, onto the set of pure states, $\tilde{\mathscr{S}}$, such that, for $p \in \widetilde{\mathscr{E}}, \alpha_{p}$ is the unique state $\alpha \in \mathscr{S}$ with $P(p, \alpha)=1$.

Moreover, if Axiom I.9 is satisfied and $p \in \tilde{\mathscr{E}}$, then $p$ is the support of $\alpha_{p}$.

Proof. First, consider part b) of Axiom 1.9. Suppose $\alpha \in \mathscr{S}$ and $p \in \mathscr{E}$ has the following property: for $\beta \in \mathscr{S}, P(p, \beta)=1$ if and only if $\beta=\alpha$. Since $P(p, \alpha)=1$, one has $p_{\alpha} \leqq 1$; consequently for $\beta \in \mathscr{S}$, $P\left(p_{\alpha}, \beta\right)=1$ implies $P(p, \beta)=1$ and, hence, $\beta=\alpha$. Conversely, if $\beta \in \mathscr{S}$ and $\beta=\alpha$, then $P\left(p_{\alpha}, \beta\right)=P\left(p_{\alpha}, \alpha\right)=1$. Consequently, part b) of Axiom I.9 is equivalent to the following: $\alpha \in \mathscr{S}$ is pure if and only if, for $\beta \in \mathscr{S}, P\left(p_{\alpha}, \beta\right)=1$ is equivalent to $\beta=\alpha$.

Suppose $\alpha \in \mathscr{S}$ and $p_{\alpha}$ satisfies the property: (I) for $\beta \in \mathscr{S}, P\left(p_{\alpha}, \beta\right)=1$ is equivalent to $\beta=\alpha$. It is asserted first that $p_{\alpha}$ is an atom. Indeed, let $q \in \mathscr{E}, q \neq 0$, and $q \leqq p_{\alpha}$. By Axiom I.8, there exists a state $\beta \in \mathscr{S}$ such that the support $p_{\beta}$ of $\beta$ equals $q$; hence, $P\left(p_{\alpha}, \beta\right)=1$, since $p_{\beta}=q \leqq p_{\alpha}$. Therefore, $\beta=\alpha$ and $q_{\beta}=p=p_{\alpha}$; consequently, $p_{\alpha}$ is, indeed, an atom. Let $\beta \in \mathscr{S}$ and suppose the support of $\beta$ equals $p_{\alpha}$. Then $P\left(p_{\alpha}, \beta\right)$ $=P\left(p_{\beta}, \beta\right)=1$ and $\beta=\alpha$. Therefore, if $p_{\alpha}$ satisfies $(\mathrm{I})$, then $p_{\alpha}$ satisfies: (II) $p_{\alpha}$ is an atom and $\alpha$ is the unique state with support equal to $p_{\alpha}$. Suppose now that $p_{\alpha}$ satisfies (II). If $\beta \in \mathscr{S}$ and $P\left(p_{\alpha}, \beta\right)=1$, then $p_{\beta} \leqq p_{\alpha}$ and, hence, $p_{\beta}=p_{\alpha}$, since $p_{\alpha}$ is an atom; consequently, $\beta=\alpha$ since $\alpha$ is the unique state with support equal to $p_{\alpha}$. Therefore, if $p_{\alpha}$ satisfies (II), then $p_{\alpha}$ satisfies (I).

Consequently, a) and b) are equivalent. Assume now the validity of b) for $(\mathscr{E}, \mathscr{S}, P) . \mathscr{E}$ is atomic provided: if $p \in \mathscr{E}$ and $p \neq 0$, then there exists an atom $q \leqq p$. If $p \in \mathscr{E}$ and $p \neq 0$, then there exists a pure state $\alpha \in \mathscr{S}$ such that $P(p, \alpha)=1$; consequently, $p_{\alpha}$ is an atom such that $p \alpha \leqq p$. Therefore, $\mathscr{E}$ is atomic. If $p \in \widetilde{E}$, then select a pure state $\alpha \in \mathscr{S}$ such that $P(p, \alpha)=1$. Since $p$ is an atom and $p_{\alpha} \leqq p, p$ coincides with the support of $\alpha$; consequently, there exists exactly one $\alpha \in \mathscr{S}$ such that $P(p, \alpha)=1$. Denote this $\alpha$ by $\alpha_{p}$. The mapping $p \rightarrow \alpha_{p}$ from $\tilde{\mathscr{E}}$ into $\tilde{\mathscr{S}}$ 
is surjective; indeed, if $\alpha \in \mathscr{S}$ is pure, then $\alpha=\alpha_{p}$ where $p$ is the support of $\alpha$. Consequently, b) implies c).

Assume the validity of c). If $p \in \widetilde{E}$, then $p$ is the support of $\alpha_{p}$. Indeed, $P\left(p, \alpha_{p}\right)=1$ implies $p_{\alpha} \leqq p$ and, hence, $p_{\alpha}=p$, since $p$ is an atom. Suppose $p \in \mathscr{E}$ and $p \neq 0$. Since $\mathscr{E}$ is atomic, there exists an atom $q \in \tilde{\mathscr{E}}$ such that $q \leqq p$. The pure state $\alpha_{g}$ satisfies $P\left(p, \alpha_{g}\right)=1$ since $q$ is the support of $\alpha_{g}$ and $q \leqq p$. Therefore, c) implies b). Q.E.D.

Consequently, for an event-state structure $(\mathscr{E}, \mathscr{S}, P)$ satisfying Axiom I.8, Theorem II.1 asserts that Axiom I.9 is satisfied if and only if $\mathscr{E}$ is atomic and the restriction of the mapping $\alpha \rightarrow p_{\alpha}$ from $\mathscr{S}$ onto $\mathscr{E}$ to the set of pure states, $\tilde{\mathscr{S}}$, is a one-to-one mapping of $\tilde{\mathscr{S}}$ onto the set of atoms, $\widetilde{\mathscr{E}}$, such that if $\alpha \in \tilde{\mathscr{S}}$ and $\beta \in \mathscr{S}$ with $p_{\alpha}=p_{\beta}$, then $\beta=\alpha$.

The event-state structure $(\mathscr{P}(H), \mathscr{S}, P)$ of von Neumann's Hilbert space model of quantum mechanics satisfies Axiom I.9.

Example II.1. The atoms of $\mathscr{P}(H)$ are the projections with onedimensional range. A state $\alpha \in \mathscr{S}$ is pure if and only if the density operator $D_{\alpha}$ is a projection with one-dimensional range [10]; therefore, the support of a pure state $\alpha$ is the atom $D_{\alpha}$.

\section{Semimodularity and Pure Operations}

The recent interest in the quantum logic approach to the foundations of quantum physics, at least partially, stems from the recognition of the desirability of an axiomatic characterization of von Neumann's Hilbert space model for quantum mechanics. Ideally, a criterion for the adoption of each axiom of this characterization would be the existence of a phenomenological interpretation of the axiom. The currently available "concrete representation theorems," the identification of $\left(\mathscr{E}, \leqq,^{\prime}\right)$ with an appropriate lattice of subspaces of a vector space (although not necessarily a Hilbert space), depend upon hypothesizing the atomicity and semimodularity of $\left(\mathscr{E}, \leqq,^{\prime}\right)$ (see [10], [11], [12], and [13]). The purpose of this section is to provide a direct phenomenological interpretation of the property of semimodularity when Axioms I.8 and I.9 are satisfied.

Although von Neumann's early papers on quantum mechanics involved the quantum logic approach (see [19] and [20], pp. 247-254), the first formalization of the quantum logic approach was contained in the classic work by BIRKHoff and von NEuMANN [2]. The hypothesis of modularity was imposed on the logic, $\left(\mathscr{E}, \leqq,^{\prime}\right)$, of quantum mechanics in [2] despite the fact that $\left(\mathscr{P}(H), \leqq,^{\prime}\right)$ is not modular for a separable infinite dimensional complex Hilbert space $H$. More recently, it has been recognized that the orthomodularity of $\left(\mathscr{E}, \leqq,^{\prime}\right)$ is adequate to replace modularity for many purposes; indeed, the orthomodularity of $\left(\mathscr{E}, \leqq,^{\prime}\right)$ 
is a consequence of the axioms for an event-state structure and possesses a phenomenological interpretation even when only the set of events is considered [13]. Definition A.4 and Theorems A.7 and A.8 of the Appendix indicate the interdependence of modularity, semimodularity, orthomodularity, and distributivity.

The semimodularity of $\left(\mathscr{E}, \leqq,^{\prime}\right)$ will be discussed in terms of pure operations [9].

Definition $I I I .1$. If $(\mathscr{E}, \mathscr{S}, P, \Omega)$ is an event-state-operation structure, then $x \in S_{\Omega}$ is a pure operation provided: if $\alpha \in \mathscr{D}_{x}$ and $\alpha$ is a pure state, then $x \propto$ is a pure state.

Theorem III.1. Let $(\mathscr{E}, \mathscr{S}, P, \Omega)$ be an event-state-operation structure satisfying Axioms I.8 and I.9. If $p \in \mathscr{E}$ is an atom, $\alpha \in \mathscr{S}$ and $P(p, \alpha) \neq 0$, then $\Omega_{p} \alpha=\alpha_{p}$; hence, $\Omega_{p}$ is a pure operation.

Proof. By Axiom II.3 of [15], $P\left(p, \Omega_{p} \alpha\right)=1$; since $p$ is an atom, $\Omega_{p} \alpha=\alpha_{p}$ by assertion c) of Theorem II.1. $\alpha_{p}$ is a pure state; hence, $\Omega_{p}$ is trivially a pure operation. Q.E.D.

A first indication of a connection between semimodularity and pure operations is contained in the following theorem.

Theorem III.2. Let $(\mathscr{E}, \mathscr{S}, P, \Omega)$ be an event-state-operation structure satisfying Axioms I.8 and I.9. If $\left(\mathscr{E}, \leqq,^{\prime}\right)$ is semimodular, then $\Omega_{p}$ is a pure operation for every $p \in \mathscr{E}$; hence, every $x \in S_{\Omega}$ is a pure operation.

Proof. If $p \in \mathscr{E}$ and $\alpha \in \mathscr{D}_{\Omega_{p}}$, then $P(p, \alpha) \neq 0$. Consequently, by the definition of support, $p_{\alpha} \not p$. The support $p_{\beta}$ of $\beta=\Omega_{p} \alpha$ satisfies

$$
p_{\beta} \leqq\left(p_{\alpha} \vee p^{\prime}\right) \wedge p
$$

because of Theorem I.2. If, moreover, $\alpha$ is a pure state, then $p_{\alpha}$ is an atom since Axiom I.9 holds. Since $\left(E, \leqq,^{\prime}\right)$ is atomic, orthomodular and semimodular, $\left(p_{\alpha} \vee p^{\prime}\right) \wedge p$ is an atom (see Theorem A.8 of the appendix). $p_{\beta}$ is the support of a state; hence, $p_{\beta} \neq 0$. Therefore, the support $p_{\beta}$ of $\beta=\Omega_{p} \alpha$ is an atom,

$$
p_{\beta}=\left(p_{\alpha} \vee p^{\prime}\right) \wedge p .
$$

Consequently, $\beta$ is a pure state and $\Omega_{p}$ is a pure operation. If $x \in S_{\Omega}$, then there exist $p_{1}, p_{2}, \ldots, p_{n} \in \mathscr{E}$ such that

$$
x \propto=\Omega_{p_{1}}\left(\Omega_{p_{2}}\left(\ldots \Omega_{p_{n}} \propto \ldots\right)\right)
$$

for all $\alpha \in \mathscr{D}_{x}$; therefore, if $\alpha \in \mathscr{D}_{x}$ is pure, then $x \alpha$ is pure. Q.E.D.

The crucial step in the proof of Theorem III.2 was noticing the fact that the semimodularity of the atomic, orthomodular ortholattice $\left(\mathscr{E}, \leqq,^{\prime}\right)$ yields the following property of the residuated mapping $\phi_{p}$ : if $q \in \mathscr{E}, q \nvdash p$, and $q$ is an atom, then

$$
\phi_{p}(q)=\left(q \vee p^{\prime}\right) \wedge p
$$


is an atom. Indeed, this is a characterization of the semimodularity of $\left(\mathscr{E}, \leqq,^{\prime}\right)$ when $\left(\mathscr{E}, \leqq,^{\prime}\right)$ is atomic. Consequently, if the connection between operations and residuated mappings is utilized, that is, if Axiom II. 8 is assumed, then a characterization of semimodularity is obtained.

Theorem III.3. If $(\mathscr{E}, \mathscr{S}, P, \Omega)$ is an event-state-operation structure satisfying Axioms I.8, I.9 and II.8, then the following are equivalent:

a) $(\mathscr{E}, \leqq, ')$ is semimodular,

b) $\Omega_{p}$ is a pure operation for every $p \in \mathscr{E}$; and

c) every $x \in S_{\Omega}$ is a pure operation.

Proof. b) and c) are equivalent obviously and a) implies b) by Theorem III.2 (without the assumption of Axiom II.8). Assume $\Omega_{p}$ is a pure operation for every $p \in \mathscr{E}$. To prove $\left(\mathscr{E}, \leqq,^{\prime}\right)$ is semimodular it suffies to prove (see Theorem A.8 of the Appendix): if $p \in \mathscr{E}, q \in \mathscr{E}$ and $q$ is an atom with $q * p$, then

$$
\phi_{p}(q)=\left(q \vee p^{\prime}\right) \wedge p
$$

is an atom. Consider the state $\alpha_{\chi}$ corresponding to the atom $q$. Since $p \nprec q$ and $q$ is the support of $\alpha_{q}, P\left(p, \alpha_{q}\right) \neq 0$ and $\alpha_{q}$ is in the domain of $\Omega_{p} ;$ moreover, $\alpha_{q}$ is a pure state. Since $\Omega_{p}$ is a pure operation, $\beta=\Omega_{p}\left(\alpha_{q}\right)$ is a pure state and the support $p_{\beta}$ is an atom. By Axiom II.8,

$$
p_{\beta}=\phi_{p}\left(p_{\alpha_{q}}\right)=\phi p(q)=\left(q \vee p^{\prime}\right) \wedge p
$$

and $\phi_{p}(q)$ is an atom. Q.E.D.

The rule for the phenomenological interpretation of operations presented in [15] provides a heuristic reason for asserting that $\Omega_{p}$ should be a pure operation for each $p \in \mathscr{E}$. The phenomenological characterization of the fact that a state $\alpha \in \mathscr{S}$ is pure is the following indecomposability of the corresponding ensemble. The ensemble of physical systems prepared by a state-preparation procedure corresponding to $\alpha$ can not be decomposed into two subensembles prepared by state-preparation procedures corresponding to two distinct states $\alpha_{1}$ and $\alpha_{2}$ with the following property: a system of the ensemble corresponding to $\alpha$ may be attributed to the ensemble corresponding to $\alpha_{1}$ with probability $t$ and to the ensemble corresponding to $\alpha_{2}$ with probability $1-t$, where $0<t<1$. The ensemble corresponding to $\Omega_{p} \alpha$ is constructed by selecting the systems of the ensemble corresponding to $\alpha$ for which the observation procedure for $p$ indicates that the event $p$ occurs. It is, therefore, plausible that this ensemble is indecomposable provided the ensemble corresponding to $\alpha$ is indecomposable.

\section{Summary}

If $(\mathscr{E}, \mathscr{S}, P, \Omega)$ is an event-state-operation structure, then the set $S_{\Omega}$ of operations admits the structure of a Baer *-semigroup, $\left(S_{\Omega}, \circ,{ }^{*},{ }^{\prime}\right)$. Since $\left(\mathscr{E}, \leqq,^{\prime}\right)$ is an orthomodular ortholattice, the $\operatorname{set} S(\mathscr{E})$ of residuated 
mappings of $\left(\mathscr{E}, \leqq,^{\prime}\right)$ also admits the structure of a Baer *-semigroup, $\left(S(\mathscr{E}), \circ,,^{*}\right)$. Theorem 1.1 indicated a connection between these two Baer *-semigroups: if $p \in \beta$ and $\alpha \in \mathscr{S}$ with $P(p, \alpha) \neq 0$, then the supports $p_{\beta}$ and $p_{\alpha}$ of $\beta=\Omega_{p} \alpha$ and $\alpha$, respectively, satisfy

$$
p_{\beta} \leqq \phi_{p}\left(p_{\alpha}\right)
$$

where $\phi_{p}$ is the residuated mapping

$$
\phi_{p}(q)=\left(q \vee p^{\prime}\right) \wedge p, \quad q \in \mathscr{E} .
$$

Axiom II. 8 replaces the above inequality

by an equality

$$
p_{\beta} \leqq \phi_{p}\left(p_{\alpha}\right)
$$

$$
p_{\beta}=\phi_{p}\left(p_{\alpha}\right) \text {. }
$$

Axiom II. 8 is satisfied, for example, by a compatible logic (that is, $p$ C $q$ for every pair $p, q \in \mathscr{E}$ ) and by von Neumann's Hilbert space model for quantum mechanics. If Axiom II.8 is satisfied, the there exists a homomorphism $\theta: S_{\Omega} \rightarrow S(\mathscr{E})$ of the Baer *-semigroup $\left(S_{\Omega}, \circ, *,,^{\prime}\right)$ of operations into the Baer *-semigroup $\left(S(\mathscr{E}), \circ, *,{ }^{*}\right)$ of residuated mappings. The operation $x \in S_{\Omega}$ maps states while the residuated mapping $\theta_{a}$ maps supports of states, specifically, if $\alpha \in \mathscr{D}_{x}$, then

$$
\theta_{x}\left(p_{\alpha}\right)=p_{x \alpha}
$$

where $p_{\alpha}$ and $p_{x \alpha}$ are the supports of $\alpha$ and $x \alpha$, respectively.

When the logic $\left(\mathscr{E}, \leqq,^{\prime}\right)$ is atomic and there exists a correspondence between pure states and atoms, Theorems III.2 and III.3 provide a connection between semimodularity of $\left(\mathscr{E}, \leqq,^{\prime}\right)$ and pure operations (operations which map pure states into pure states). Indeed, if $\left(\mathscr{E}, \leqq,^{\prime}\right)$ is semimodular, then every $x \in S_{\Omega}$ is a pure operation. When Axiom II.8 is imposed, $\left(\mathscr{E}, \leqq,^{\prime}\right)$ is semimodular if and only if every $x \in S_{\Omega}$ is a pure operation.

\section{Appendix}

This Appendix is devoted to the exposition of facts about the residuated mappings of an orthomodular ortholattice and about the semimodularity of lattices, specifically, atomic orthomodular ortholattices, and of the proof of one theorem for general Baer *-semigroups.

\section{A.I. Residuated Mappings}

Some definitions and theorems from the theory of posets will be needed [1, 3].

Definition $A .1$. Let $(X, \leqq)$ be a poset.

a) If $Y$ is a subset of $X$, then $x \in Y$ is a least (respectively, greatest) element for $Y$ provided: $x \leqq y$ (respectively, $y \leqq x$ ) for every $y \in Y$. (There exists at most one least (respectively, greatest) element of $Y$.) 
b) A mapping $\phi: X \rightarrow X$ is isotone provided: if $x, y \in X$ and $x \leqq y$, then $\phi(x) \leqq \phi(y)$.

c) A mapping $\phi: X \rightarrow X$ is residuated provided:

i) $\phi$ is isotone, and

ii) if $x \in X$, then the subset

$$
\{y \in X: \phi(y) \leqq x\}
$$

is nonempty and possesses a greatest element.

d) $S(X)$ denotes the set of all residuated mappings of $X$.

e) If $\phi: X \rightarrow X$ and $\psi: X \rightarrow X$, then the mapping $\phi \circ \psi: X \rightarrow X$ is defined by

for all $x \in X$.

$$
(\phi \circ \psi)(x)=\phi(\psi(x))
$$

f) If $\phi \in S(X)$, then the mapping $\phi^{+}: X \rightarrow X$ is defined as follows: for $x \in X, \phi(x)$ is the greatest element of the subset

of $X$.

$$
\{y \in X: \phi(y) \leqq x\}
$$

Theorem A.1. Let $(X, \leqq)$ be a poset.

a) An isotone mapping $\phi: X \rightarrow X$ is residuated if and only if there exists an isotone mapping $\psi: X \rightarrow X$ such that

and

$$
(\psi \circ \phi)(x) \geqq x
$$

$$
(\phi \circ \psi)(x) \leqq x
$$

for all $x \in X$; moreover, if $\phi$ is residuated, then $\psi$ is uniquely determined, $\psi=\phi^{+}$.

b) If $\phi, \psi \in S(X)$, then $\phi \circ \psi \in S(X)$ and

$$
(\phi \circ \psi)^{+}=\psi^{+} \circ \phi^{+} \text {. }
$$

c) Let 0 be a least element for $(X, \leqq), \phi \in S(X)$ and $x \in X . \phi(x)=0$ if and only $x \leqq \phi^{+}(0)$.

d) If $(X, \leqq)$ has least and greatest elements, 0 and 1 , respectively, then the mappings $0: X \rightarrow X$ and $1: X \rightarrow X$ are residuated where

$$
\begin{array}{ll}
0(x)=0, & x \in X \\
\mathbf{l}(x)=x, & x \in X ;
\end{array}
$$

moreover, $(S(X), \circ)$ is a semigroup with zero, 0 , and unit, 1.

If $(X, \leqq)$ is not only a poset but also has an orthocomplementation. then $(S(X)$, o) admits an involution [6].

Definition A.2. If ' $: X \rightarrow X$ is an orthocomplementation of a poset $(X, \leqq)$ and $\phi \in S(X)$, then the mapping $\phi^{*}: X \rightarrow X$ is defined by

$$
\phi^{*}(x)=\phi^{+}\left(x^{\prime}\right)^{\prime}, \quad x \in X .
$$


Theorem A.2. Let ' $X \rightarrow X$ be an orthocomplementation of the poset $(X, \leqq)$

a) If $\alpha \in S(X)$ and $x \in X$, then

$$
\phi^{+}(x)=\phi^{*}\left(x^{\prime}\right)^{\prime}
$$

and $\phi^{*}(x)$ is the least element of the subset

$$
\left\{y^{\prime}: \phi(y) \leqq x^{\prime}\right\}
$$

of $X$.

b) If $\phi \in S(X)$, then $\phi^{*} \in S(X)$; moreover, $\phi^{*}$ is the unique isotone mapping $\psi: X \rightarrow X$ such that

and

$$
\psi\left(\phi\left(x^{\prime}\right)^{\prime}\right) \leqq x
$$

for all $x \in X$.

$$
\phi\left(\psi\left(x^{\prime}\right)^{\prime}\right) \leqq x
$$

c) Let $(X, \leqq)$ have least and greatest elements 0 and $1, \phi \in S(X)$, and $x \in X . \phi(x)=0$ if and only if $x \perp \phi^{*}(1)$.

d) * is an involution for the semigroup $(S(X), 0)$.

If $\left(X, \leqq,^{\prime}\right)$ is not only an orthoposet but also an orthomodular ortholattice, then $S(X)$ admits the structure of a Baer *-semigroup [6].

Definition A.3. Let $\left(L, \leqq,^{\prime}\right)$ be an ortholattice.

a) If $p \in L$, then the mapping $\phi_{p}: L \rightarrow L$ is defined by

$$
\phi_{p}(q)=\left(q \vee p^{\prime}\right) \wedge p, \quad q \in L .
$$

b) If $\phi \in S(L)$, then the mapping $\phi^{\prime}: L \rightarrow L$ is defined by $\phi^{\prime}=\phi_{p}$ where $p=\phi^{*}(1)^{\prime}$.

Theorem A.3. Let $\left(L, \leqq,^{\prime}\right)$ be an orthomodular ortholattice.

a) If $p \in L$, then $\phi_{p} \in S(L)$; moreover,

$$
\phi_{p}^{*}=\phi_{p} \circ \phi_{p}=\left(\phi_{p}\right)^{\prime \prime}=\phi_{p} .
$$

b) For $p, q \in L, \phi_{p}(q)=0$ if and only if $p \perp q$.

c) $(S(L), \circ, *, ')$ is a Baer *-semigroup such that $p \rightarrow \phi_{p}$ is an isomorphism of the orthomodular ortholattice $\left(L, \leqq,^{\prime}\right)$ onto the orthomodular ortholattice $\left(P^{\prime}(S(L)), \leqq,^{\prime}\right)$ of closed projections in $S(L)$.

\section{A.II. Two Theorems on Baer *-semigroups}

The following theorem is from the general theory of Baer *-semigroups $[6,7,8]$.

Theorem A.4. If $\left(S, \circ,{ }^{*},{ }^{\prime}\right)$ is a Baer *-semigroup, then

a) for $x \in S,\left(x^{*} \circ x\right)^{\prime \prime}=x^{\prime \prime}$,

b) for $x, y \in S,(x \circ y)^{\prime \prime}=\left(x^{\prime \prime} \circ y\right)^{\prime \prime}$,

c) for $e, f \in P^{\prime}(S),(e \circ f)^{\prime \prime}=\left(e \vee f^{\prime}\right) \wedge f$. 
The computation of the operator-theoretic support projection of $P D P$, where $D$ is a positive operator and $P$ is a projection on a Hilbert space $H$, is an application of the following theorem to the Baer *-semigroup $\left(\mathscr{L}_{c}(H), \circ, *,,^{\prime}\right)$ of continuous linear operators on $H$.

Theorem A.5. If $\left(S, \circ,{ }^{*},{ }^{\prime}\right)$ is a Baer *-semigroup, $y \in S, e \in P^{\prime}(X)$ and $x=y^{*} \circ y$, then $(e \circ x \circ e)^{\prime \prime}=\left(x^{\prime \prime} \vee e^{\prime}\right) \wedge e$.

Proof. First, one notes

$$
e \circ x \circ e=(y \circ e)^{*} \circ(y \circ e)
$$

and, by a) of Theorem A.4,

$$
(e \circ x \circ e)^{\prime \prime}=(y \circ e)^{\prime \prime} .
$$

Utilizing b) of Theorem A.4, one has

hence,

$$
(y \circ e)^{\prime \prime}=\left(y^{\prime \prime} \circ e\right)^{\prime \prime} ;
$$

$$
(e \circ x \circ e)^{\prime \prime}=\left(y^{\prime \prime} \circ e\right)^{\prime \prime} .
$$

Since $y^{\prime \prime} \in P^{\prime}(S)$ and $e \in P^{\prime}(S)$, c) of Theorem A.4 asserts

$$
\left(y^{\prime \prime} \circ e\right)^{\prime \prime}=\left(y^{\prime \prime} \vee e^{\prime}\right) \wedge e .
$$

Since $x=y^{*} \circ y$, another application of a) of Theorem A.4 yields

consequently,

$$
x^{\prime \prime}=\left(y^{*} \circ y\right)^{\prime \prime}=y^{\prime \prime} ;
$$

$$
(e \circ x \circ e)^{\prime \prime}=\left(x^{\prime \prime} \vee e^{\prime}\right) \wedge e,
$$

the assertion of the theorem. Q.E.D.

\section{A.III. Semimodularity and Atomicity}

The following definition and theorem indicate the interdependence of distributivity, orthomodularity, semimodularity, and modularity $[1,11]$.

Definition A.4. Let $(L, \leqq)$ be a lattice.

a) If $p, q, r \in L$, then $(p, q, r)$ is a distributive triple, written $(p, q, r) D$, provided:

$$
(p \vee q) \wedge r=(p \wedge r) \vee(q \wedge r) .
$$

$(L, \leqq)$ is a distributive lattice provided: if $p, q, r \in L$, then $(p, q, r) D$.

b) If $q, r \in L$, then $(q, r)$ is a modular pair, written $(q, r) M$, provided: if $p \in L$ and $p \leqq r$, then $(p, q, r) D$. ( $L, \leqq)$ is a modular lattice provided: if $p, q \in L$, then $(p, q) M$.

c) $(L, \leqq)$ is semimodular provided: if $p, q \in L$ and $(p, q) M$, then $(q, p) M$.

Theorem A.6. An ortholattice $\left(L, \leqq,^{\prime}\right)$ is orthomodular if and only if every orthogonal pair is a modular pair, i.e., $p, q \in L$ and $p \perp q$ implies $(p, q) M$. 
Consequently, every distributive lattice is modular and every modular ortholattice is orthomodular. It should be noted that the projection lattice of every von Neumann algebra is not only orthomodular but also semimodular [18]. Semimodularity admits a useful characterization in an atomic orthomodular ortholattice [17].

Definition A5. Let $(X, \leqq)$ be a poset with a least element 0 .

a) An element $x \in X$ is an atom provided:

i) $x \neq 0$ and

ii) if $y \in X$ and $y \leqq x$, then either $y=0$ or $y=x$.

b) $(X, \leqq)$ is atomic provided: if $x \in X$ and $x \neq 0$, then there exists an atom $y \in X$ such that $y \leqq x$.

Theorem A.7. A necessary and sufficient condition for an atomic orthomodular ortholattice ( $\left.L, \leqq,^{\prime}\right)$ to be semimodular is the following: if $p \in L$, $q \in L$ is an atom and $q$ is not orthogonal to $p, q \nless p$, then $\left(q \vee p^{\prime}\right) \wedge p$ is an atom.

Acknowledgements. The author expresses his gratitude to A. B. RAMSAY for a continuing dialogue on the mathematical foundations of quantum physics, to J. M. JAUCH, C. PrRon, and J. Sopka for numerous informative discussions, and to J. M. JAUCH for the hospitality of the Institut de Physique Théorique de l'Université de Genève.

\section{References}

1. Birknoff, G.: Lattice theory, American Mathematical Society Colloquium Publications, Vol. 25, 3rd. Ed., Providence, R. I. (1967).

2. - , and J. von Neumann: The logic of quantum mechanics. Ann. Math. 37, $823-843(1936)$.

3. Derderian, J. C.: Residuated mappings. Pacific J. Math. 20, 35-43 (1967).

4. Dixmrer, J. : Les algebres d'operateurs dans l'espace hilbertian. Paris: GauthierVillars 1957.

5. - Les $C^{*}$-algebres et leurs representations. Paris: Gauthier-Villars 1964.

6. Foulis, D. J.: Baer *-semigroups. Proc. Am. Math. Soc. 11, 648-654 (1960).

7. - A note on orthomodular lattices. Portugal. Math. 21, 65-72 (1962).

8. - Relative inverses in Baer *-semigroups. Michigan Math. J. 10, 65-84 (1963).

9. HAAG, R., and D. KaSTLER: An algebraic approach to quantum field theory. J. Math. Phys. 5, 848-861 (1964).

10. JAUCH, J. M.: Foundations of quantum mechanics. Reading, Mass.: AddisonWesley 1968.

11. Macharen, M. D.: Atomic orthocomplemented lattices. Pacific J. Math. 14, $597-612(1964)$.

12. - Notes on axioms for quantum mechanics. Argonne National Laboratory Report, ANL-7065 (1965).

13. Prron, C.: Axiomatique quantique. Helv. Phys. Acta 37, 439-468 (1964).

14. Pool, J. C. T.: Simultaneous observability and the logic of quantum mechanics. Thesis, State University of Iowa, Department of Physics Report SUI-63-17 (1963).

15. - Baer *-semigroups and the logic of quantum mechanics, Commun. Math. Phys. 9, 118-141 (1968). 
16. Schatten, R.: Norm ideals of completely continuous operators. BerlinGöttingen-Heidelberg: Springer 1960.

17. Schreiner, E. A.: Modular pairs in orthomodular lattices. Pacific J. Math. 19, $519-528(1966)$.

18. Topping, D. M.: Asymptoticity and semimodularity in projection lattices. Pacific J. Math. 20, 317-325 (1967).

19. von Neumann, J.: Wahrscheinlichkeitstheoretischer Aufbau der Quantenmechanik. Nachr. Akad. Wiss. Göttingen Math.-Phys. Kl. II, 245-272 (1927).

20. - Mathematical foundations of quantum mechanics. Trans. by R. T. Beyer. Princeton: Princeton Univ. Press. 1955.

21. Zierler, N.: Axioms for non-relativistic quantum mechanics. Pacific J. Math. 11, 1151-1169 (1761).

J. C. T. Pool

Department of Mathematics

University of Massachusetts

Amherst, Massachusetts 01002, USA 